\title{
CO OBSERVATIONS OF LUMINOUS IR GALAXY MERGERS
}

\author{
Y. GAO \\ Dept. of Astronomy, University of Illinois, Urbana, IL \\ AND \\ P.M. SOLOMON \\ Dept. of Phy. \& Astronomy, SUNY at Stony Brook, NY
}

Luminous starbursts are observed to occur mostly as a result of a collision/merger in gas-rich galaxies, and most luminous infrared galaxies (LIGs) are indeed gas-rich mergers. In order to determine the relationship between the IR and molecular gas properties and the galaxy-galaxy interactions, we study LIG mergers in the intermediate merging process. We have observed nearly 20 LIG mergers and together with the CO data in the literature, we have found a correlation between the CO luminosity, $L_{\mathrm{CO}}$, and the projected separation of merger nuclei, $R_{\mathrm{Sep}}$, in $>50$ LIG mergers. The correlation suggests the molecular content is decreasing as merging advances and is better established with $\sim 40$ LIG mergers excluding ultraluminous ones, which resembles more a volume-limited, statistically complete sample of LIG mergers. In addition, an anti-correlation between $L_{\mathrm{IR}} / L_{\mathrm{CO}}$ (the measure of star formation efficiency, SFE) and $R_{\mathrm{Sep}}$ is evident. One interpretation is that the molecular gas content of LIG mergers is being rapidly depleted due to the merger-induced starbursts and the increase of SFE as merging progresses.

Both numerical simulations and observations have shown the vital role of the gas in triggering the starburst in galaxy mergers. Correlations we found here do suggest a sequence of merging can be traced by studying the molecular ISM in LIGs. This is because LIG mergers have roughly comparable initial gas reservoirs since they are all mergers of gas-rich spirals and the ultraluminous starburst phase can eventually be reached. In comparison, the "Toomre sequence" or optically selected samples of interacting pairs are mixtures of various gas content and many of them will never reach the ultraluminous phase and the merger sequence in terms of the gas content and the peak starburst (ultraluminous) phase is not well represented. 


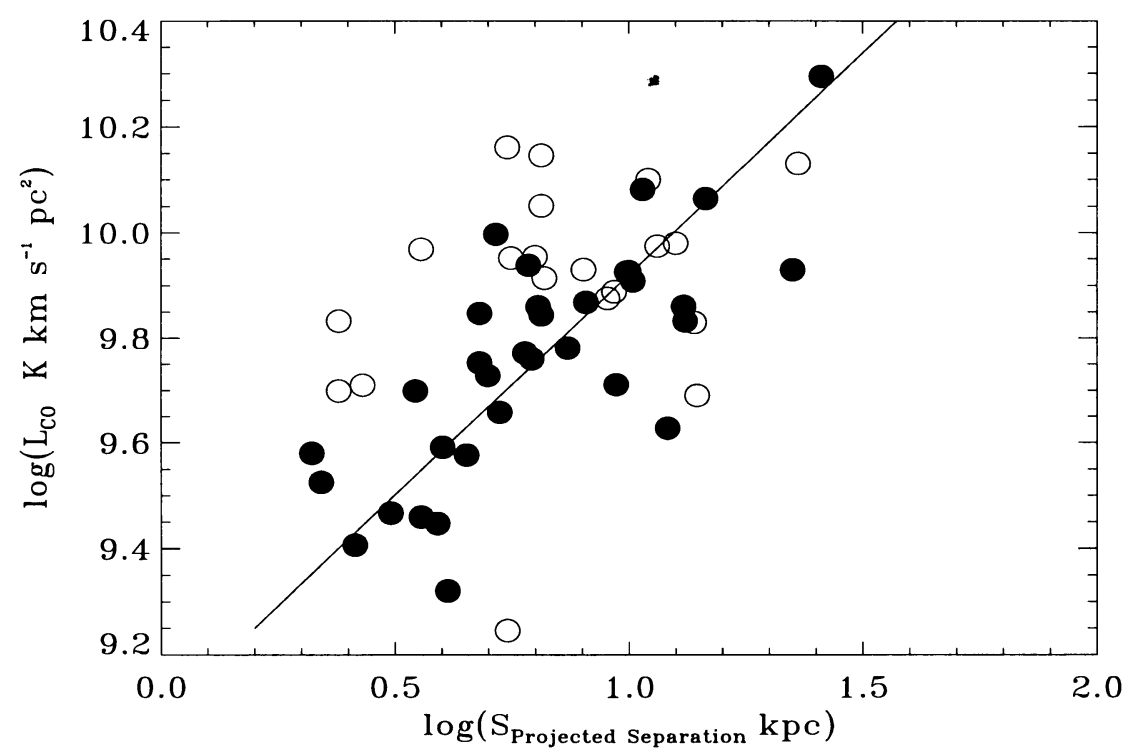

Figure 1. The correlation between $L_{\mathrm{CO}}$ and $R_{\mathrm{Sep}}$ for $>50$ LIG mergers imlies molecular gas mass decreases as merging progresses. Open circles are for ultraluminous mergers

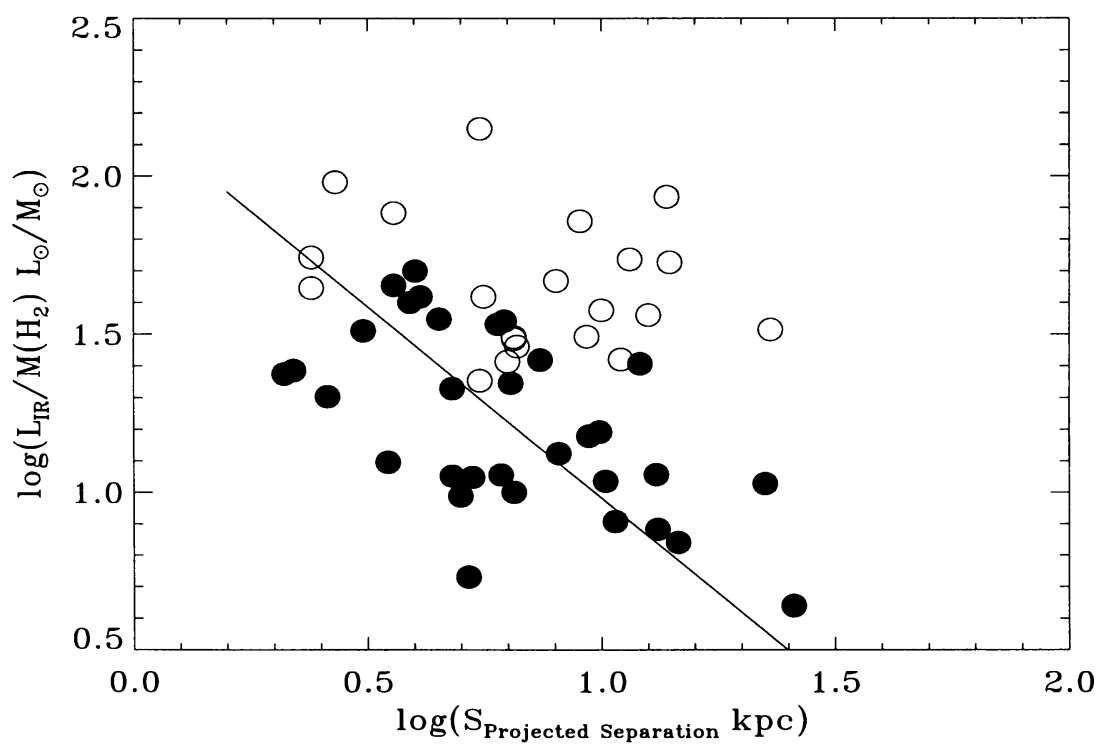

Figure 2. The anti-correlation between $L_{\mathrm{IR}} / L_{\mathrm{CO}}(\mathrm{SFE})$ and $R_{\mathrm{Sep}}$ suggests enhanced SFE as merging advances. 